\title{
Mean Commute Time for Random Walks on Hierarchical Scale-Free Networks
}

\author{
Yilun Shang
}

Abstract. In recent years, there has been a surge of research interest in networks with scale-free topologies, partly due to the fact that they are prevalent in scientific research and real-life applications. In this paper, we study random-walk issues on a family of two-parameter scale-free networks, called $(x, y)$-flowers. These networks, which are constructed in a deterministic recursive fashion, display rich behaviors such as the small-world phenomenon and pseudofractal properties. We derive analytically the mean commute times for random walks on $(x, y)$-flowers and show that the mean commute times scale with the network size as a power-law function with exponent governed by both parameters $x$ and $y$. We also determine the mean effective resistance and demonstrate that it changes sharply between different choices of $x$ and $y$. Furthermore, we compare mean commute times for $(x, y)$-flowers with those for Erdős-Rényi random graphs. Our theoretical results are verified by numerical studies.

\section{Introduction}

As one of the most studied stochastic processes, random walks [Lovász 96, Weiss 94] have attracted considerable research attention due to their widespread

(C) Taylor \& Francis Group, LLC

ISSN: 1542-795I print 
application in various theoretical and applied fields, including statistical physics [Burioni and Cassi 05, Metzler and Klafter 00, Shang 10], computational biology [Codling et al. 08], machine vision [Meila and Shi 01, Turk 01], and structural pattern analysis [Fouss et al. 07, Gori et al. 04]. Random walks, for instance, are the basis of the PageRank algorithm used by the Google bot search engine [Brin and Page 98]. It is also well known that there is a basic correspondence between random walks on graphs and electrical networks [Doyle and Snell 84]. Among a number of important quantities related to random walks, the commute time $C_{u v}$ of a random walk on a graph is of particular interest. It is defined as the expected number of steps for the random walk to travel from node $u$ to node $v$ and then return to $u$. In the last few years, the commute time has received increasing attention, since it can be used as a robust measure of network search efficiency [Bartumeus and Levin 08, García Cantú and Abad 08, Maiya and Berger-Wolf 10, Tejedor et al. 11], proximity of data, and cluster cohesion [Esfandiar et al. 10, Qiu and Hancock 07, Yen et al. 07].

Most of the existing literature on the commute time addresses random walks on some particular graphs with relatively simple structure, such as trees [Palacios 09], regular lattices [Lovász 96, Montroll 56], Sierpiński gaskets [Bentz et al. 10, Hattori et al. 94], homogeneous random graphs [Sood et al. 05], and the like. However, recent studies have revealed that many real-world complex networks such as the Internet, World Wide Web, and social networks are scale-free [Barabási and Albert 99]: the degree distribution $P(k)$ (that is, the distribution that governs the probability that a node will have degree $k$ ) follows a power law $P(k) \propto k^{-\gamma}$ with degree exponent $\gamma$ in the range $2 \leq \gamma \leq 3$. This highly inhomogeneous structural feature profoundly affects various dynamical processes taking place on networks [Bollobás and Riordan 04, Newman et al. 06, Tanaka et al. 12]. Much effort has been devoted to random-walk issues on stochastic scalefree network models (such as the Molloy-Reed scheme [Molloy and Reed 95] and Barabási-Albert model [Barabási and Albert 99]); see, e.g., [Candia et al. 07, Gallos 04, Hruz and Peter 11, Noh and Kim 06, Polynikis 06] and references therein.

In this paper, we study simple random walks on a family of deterministic scale-free networks, called $(x, y)$-flowers [Rozenfeld et al. 07, Rozenfeld and benAvraham 07]. These networks are constructed in a recursive fashion so that each network contains $x+y$ subgraphs that resemble the whole. In this sense, the $(x, y)$-flowers can be viewed as hierarchical networks [Hinczewski and Berker 06, Shang 12]. We determine analytically the mean commute time between hubs (nodes of highest degree) and other nodes for the random walks. Our result indicates that the mean commute time scales with the network size in the form of a power law, whose exponent is dictated by the two parameters $x$ and $y$. The corresponding mean effective resistance is shown to change sharply from zero to 
infinity between different combinations of parameters in the limit of large network size. One interesting finding is that $(x, y)$-flowers with $x>1$ possess longer mean commute times even than sparse Erdős-Rényi graphs with the same size and a logarithmically diverging average degree $\langle k\rangle=\Theta(\ln n)$. Numerical calculations are performed to test our theoretical results.

To the best of our knowledge, there are no existing studies on commute time or effective resistance in $(x, y)$-flowers. We should mention some prior work that is conceptually or spiritually relevant. The average trapping time for random walks with a fixed trap on $(x, y)$-flowers is analyzed in [Zhang et al. 09, Zhang et al. 11] by numerical or analytical means. Bond percolation processes on $(x, y)$-flowers are investigated in [Rozenfeld and ben-Avraham 07], where percolation thresholds are derived using renormalization group techniques. In [Hinczewski and Berker 06], the authors consider the critical behavior of the ferromagnetic Ising model on some limiting cases of $(x, y)$-flowers. Numerical studies of reactiondiffusion processes and susceptible-infected (SI) epidemic processes running over $(x, y)$-flowers are conducted in [Yun et al. 09] and [Chu et al. 10], respectively.

The rest of this paper is organized as follows. In Section 2, we present the network model as well as some basic properties. The mean commute time is derived in Section 3. We conclude the paper in Section 4 with some open problems.

\section{Hierarchical Scale-Free Networks}

The hierarchical scale-free networks called $(x, y)$-flowers are constructed in a recursive fashion [Rozenfeld et al. 07, Rozenfeld and ben-Avraham 07]. Each edge in generation $n$ is replaced by two parallel paths consisting of $x$ and $y$ edges, to yield generation $n+1$; see Figures 1 and 2. In what follows we assume that $x \leq y$ and $y>1$, without loss of generality. Denote by $F_{n}(x, y)$ the $(x, y)$ flowers in generation $n$. $F_{0}(x, y)$ consists of two initial nodes connected by an edge, and $F_{1}(x, y)$ is a cycle consisting of $x+y \equiv z$ edges and nodes. The special case of $x=1$ and $y=2$ (cf. Figure 1) was promoted by [Dorogovtsev et al. 02] a decade ago.

From the method of construction, it is easy to derive that the numbers of edges and nodes in $F_{n}(x, y)$, the $(x, y)$-flowers of generation $n$, are

$$
M_{n}=(x+y)^{n}=z^{n}
$$

and

$$
N_{n}=\left(\frac{z-2}{z-1}\right) z^{n}+\frac{z}{z-1}
$$




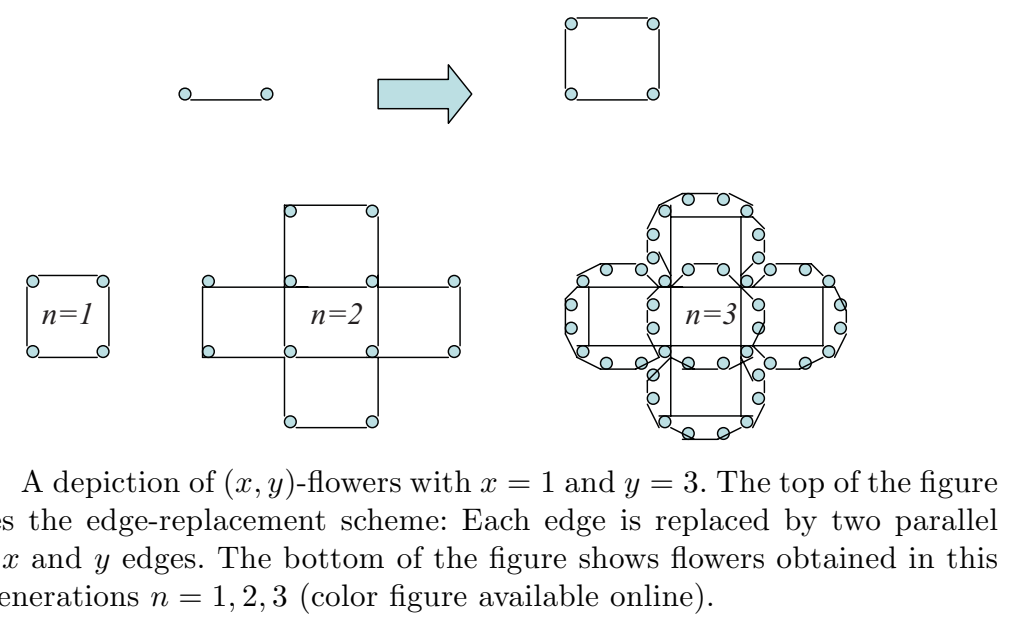

respectively. We note that $(x, y)$-flowers with the same parameter $z$ have the same degree sequence and hence identical degree distribution [Rozenfeld et al. 07]. In addition, they have been shown to have degree distribution of the form $P(k) \propto k^{-\gamma}$, with $\gamma=1+\ln z / \ln 2$, and are thus scale-free. In both $(1,3)$ - and $(2,2)$-flowers, for example, we have $\gamma=1+\ln 4 / \ln 2=3$. Considering shortest paths, $(x, y)$-flowers with $x=1$ are small worlds and are otherwise fractals with fractal dimension (in chemical space) $d_{f}=\ln z / \ln x$ [Rozenfeld et al. 07]. These and other topological properties, such as assortativity and clustering, make them a viable model for real-life networks associated with some complex

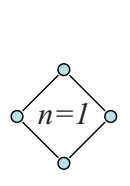

O
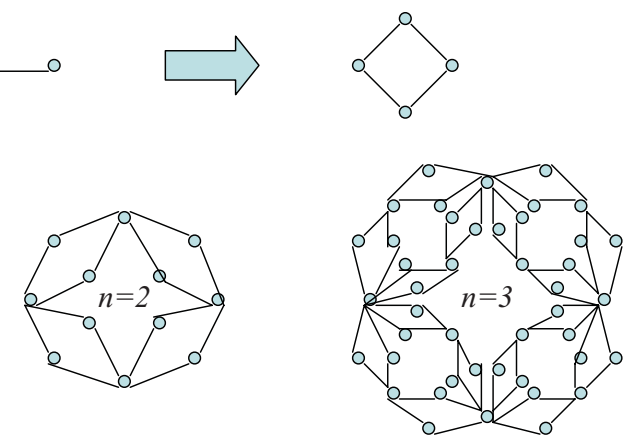

Figure 2. A depiction of $(x, y)$-flowers with $x=2$ and $y=2$. The top of the figure illustrates the edge-replacement scheme: Each edge is replaced by two parallel paths of $x$ and $y$ edges. The bottom of the figure shows flowers obtained in this way to generations $n=1,2,3$ (color figure available online). 
systems [Rozenfeld and ben-Avraham 07], while their self-similarity makes them amenable to rigorous mathematical treatment.

\section{Random Walks on $(x, y)$-Flowers}

In this section, we investigate the simple discrete-time random walks of a walker on the network $F_{n}(x, y)$. At each time step, the walker moves from its current location to one of its neighbor nodes with equal probability [Lovász 96]. The commute time $C_{u v}$ between nodes $u$ and $v$ is the expected number of steps in a random walk commencing from node $u$, reaching node $v$, and then returning to $u$. In $(x, y)$-flowers, the two initial nodes, which will be denoted by $i_{0}$ and $j_{0}$, are the most-connected (i.e., hub) nodes in the graph. We will focus on the commute time between $i_{0}$ and any other node, say $v$, in $F_{n}(x, y)$, namely, the expected time in a random walk starting at $i_{0}$, reaching $v$, and then returning to $i_{0}$. We denote this commute time by $C_{i_{0} v}^{(n)}$.

Let $V_{n}$ be the vertex set of $(x, y)$-flower $F_{n}(x, y)$. By definition, we have $N_{n}=$ $\left|V_{n}\right|$. The mean commute time, $\langle C\rangle_{n}$, which is the average of $C_{i_{0} v}^{(n)}$ over all nodes in $F_{n}(x, y)$, is given by

$$
\langle C\rangle_{n}=\frac{1}{N_{n}} \sum_{v \in V_{n}} C_{i_{0} v}^{(n)} .
$$

Clearly, for all $n$ we have $C_{i_{0} i_{0}}^{(n)}=0$. Since $F_{1}(x, y)$ is a cycle, it is easy to check that $C_{i_{0} j_{0}}^{(1)} / 2=x y \equiv w$. In what follows, we will see that the parameter $w$ together with $z \equiv x+y$ plays an important role in the mean commute time for random walks on $(x, y)$-flowers.

Theorem 3.I. For a simple random walk on $F_{n}(x, y)$, we have

$$
\langle C\rangle_{n} \sim \frac{z^{2}-4}{3(w-1)} N_{n}^{\ln w / \ln z},
$$

as $n \rightarrow \infty$, where $N_{n}$ is given by (2.2) and $a_{n} \sim b_{n}$ means $a_{n} / b_{n} \rightarrow 1$ as $n \rightarrow \infty$.

Note that we have $w>1$ and $z>2$ by the assumptions $x \leq y$ and $y>1$. Thus, it follows from (3.2) that the dominating scaling of mean commute time grows as a power-law function of network size $N_{n}$ with the exponent $\ln w / \ln z$. It is easy to see that this exponent is no less than 1 for $x>1$, namely, for those $(x, y)$-flowers with finite fractal dimensions [Rozenfeld et al. 07]. It is shown in [Sood et al. 05] that in the Erdős-Rényi random graph $G(N, p)$ with $p>\ln N / N$, the commute time between any two nodes $u$ and $v$ satisfies $C_{u v} \sim 2 N$ almost surely. Owing to 
the homogeneity of Erdös-Rényi random graphs, we may let $i_{0}$ be any vertex in $G(N, p)$ and define $\langle C\rangle$ as the average of $C_{i_{0}}$ over all nodes in $G(N, p)$. Clearly, the relation $\langle C\rangle \sim 2 N$ still holds. The above discussion leads to the following corollary.

Corollary 3.2. Let the $(x, y)$-flower $F_{n}(x, y)$ have the same size as the Erdös-Rényi random graph $G(N, p)$, i.e., $N_{n}=N$. The following statements are true as $n \rightarrow$ $\infty$ and $N \rightarrow \infty$ :

- The mean commute times in $(x, y)$-flowers with $x=1$, i.e., small-world graphs and nonfractals, are less than those in connected Erdös-Rényi random graphs almost surely.

- The mean commute times in $(x, y)$-flowers with

$$
x>1 \text { and }(x-2)^{2}+(y-2)^{2}>0,
$$

i.e., fractals, are greater than those in connected Erdös-Rényi random graphs almost surely.

- The mean commute times in $(2,2)$-flowers are asymptotically equal to those in connected Erdös-Rényi random graphs almost surely.

If we define the average degree of $F_{n}(x, y)$ as

$$
\langle k\rangle_{n}=\frac{2 M_{n}}{N_{n}}=\frac{2(z-1) z^{n-1}}{(z-2) z^{n-1}+1},
$$

which tends to $2(z-1) /(z-2)$, then by making use of Theorem 3.1 , we may obtain an asymptotic upper bound in terms of average degree $\langle k\rangle_{n}$.

Corollary 3.3. We have

$$
\begin{aligned}
\langle C\rangle_{n} & \sim \frac{8\left(2\langle k\rangle_{n}-3\right)}{3\left(\langle k\rangle_{n}-2\right)^{2}\langle k\rangle_{n}(w-1)}\left(\frac{2\left(\langle k\rangle_{n}-1\right)}{\langle k\rangle_{n}-2}\right)^{n \ln w / \ln z} \\
& \leq \frac{4\left(2\langle k\rangle_{n}-3\right)}{3\langle k\rangle_{n}\left(\langle k\rangle_{n}-2\right)}\left(\frac{2\left(\langle k\rangle_{n}-1\right)}{\langle k\rangle_{n}-2}\right)^{2 n \ln \left(\frac{\langle k\rangle_{n}-1}{\langle k\rangle_{n}-2}\right) / \ln \left(\frac{2\left(\langle k\rangle_{n}-1\right)}{\langle k\rangle_{n}-2}\right)},
\end{aligned}
$$

as $n \rightarrow \infty$.

Proof. From (3.3), we know that the average degree satisfies

$$
\langle k\rangle_{n} \sim \frac{2(z-1)}{z-2}
$$


as $n \rightarrow \infty$. Accordingly, we have

$$
z \sim \frac{2\left(\langle k\rangle_{n}-1\right)}{\langle k\rangle_{n}-2} .
$$

Substituting (3.5) into (2.2), we derive

$$
N_{n} \sim \frac{2}{\langle k\rangle_{n}}\left(\left(\frac{2\left(\langle k\rangle_{n}-1\right)}{\langle k\rangle_{n}-2}\right)^{n}+\langle k\rangle_{n}-1\right),
$$

as $n \rightarrow \infty$.

Therefore, it follows from (3.5), (3.6), and (3.2) that

$$
\begin{aligned}
\langle C\rangle_{n} & \sim \frac{2\left(z^{2}-4\right)}{3(w-1)\langle k\rangle_{n}}\left(\frac{2\left(\langle k\rangle_{n}-1\right)}{\langle k\rangle_{n}-2}\right)^{n \ln w / \ln z} \\
& \sim \frac{8\left(2\langle k\rangle_{n}-3\right)}{3\left(\langle k\rangle_{n}-2\right)^{2}\langle k\rangle_{n}(w-1)}\left(\frac{2\left(\langle k\rangle_{n}-1\right)}{\langle k\rangle_{n}-2}\right)^{n \ln w / \ln z},
\end{aligned}
$$

as $n \rightarrow \infty$. By our definition, the inequality $z-1 \leq w \leq(z / 2)^{2}$ holds. Using this inequality in (3.7), we finally arrive at the desired upper bound (3.4).

When a graph $G=(V, E)$ is considered as an electrical network with unit conductances on the edges, it is well known [Chandra et al. 96] that $C_{u v}=$ $2|E| R_{u v}$, where $R_{u v}$ is the effective resistance between $u$ and $v$. Let $R_{u v}^{(n)}$ be the effective resistance between $u$ and $v$ in $F_{n}(x, y)$, and define the mean effective resistance as

$$
\langle R\rangle_{n}=\frac{1}{N_{n}} \sum_{v \in V_{n}} R_{i_{0} v}^{(n)}
$$

Corollary 3.4. We have

$$
\begin{aligned}
\langle R\rangle_{n} & \sim \frac{z^{2}-4}{6(w-1) z^{n}} N_{n}^{\ln w / \ln z} \\
& \rightarrow \begin{cases}0 & \text { if } x=1 \\
\infty, & \text { if } x>1 \text { and }(x-2)^{2}+(y-2)^{2}>0 \\
O(1) & \text { if } x=y=2\end{cases}
\end{aligned}
$$

as $n \rightarrow \infty$.

Proof. It follows from (3.1) and $C_{i_{0} v}^{(n)}=2 M_{n} R_{i_{0} v}^{(n)}$ that

$$
\langle C\rangle_{n}=\frac{2 M_{n}}{N_{n}} \sum_{v \in V_{n}} R_{i_{0} v}^{(n)} .
$$




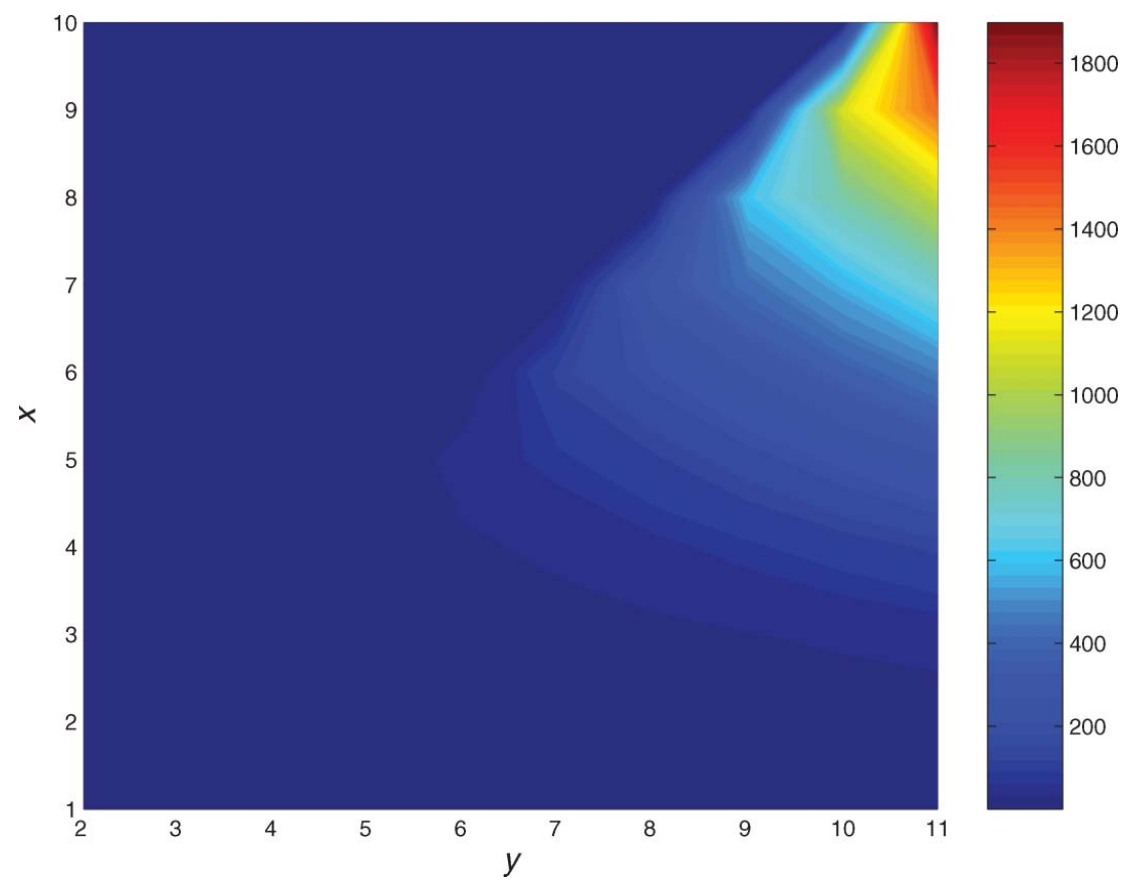

Figure 3. The plot shows the mean effective resistance of $F_{n}(x, y)$ with $n=5$ as a function of $x$ and $y$. This effective resistance, $\langle R\rangle_{5}$, is color coded as indicated in the right panel (color figure available online).

Hence for large $n$,

$$
\begin{aligned}
\langle R\rangle_{n} & =\frac{\langle C\rangle_{n}}{2 M_{n}} \sim \frac{z^{2}-4}{6(w-1) z^{n}} N_{n}^{\ln w / \ln z} \\
& \sim \frac{(z+2)(z-2)^{1+\ln w / \ln z}}{6(w-1)(z-1)^{\ln w / \ln z}} \cdot z^{n(\ln w / \ln z-1)},
\end{aligned}
$$

and using (3.8), (3.10), (2.2), and Theorem 3.1, the result follows.

In Figure 3, we plot the mean effective resistance $\langle R\rangle_{n}$ of $F_{n}(x, y)$ with $n=5$ as a function of $x$ and $y$. This effective resistance is an increasing function of both $x$ and $y$ for every $n$.

Now we turn to the proof of Theorem 3.1.

Proof of Theorem 3.I. Let $H_{u v}^{(n)}$ be the hitting time for a random walk from node $u$ to node $v$ in $F_{n}(x, y)$, that is, the expected number of steps before node $v$ is visited, 


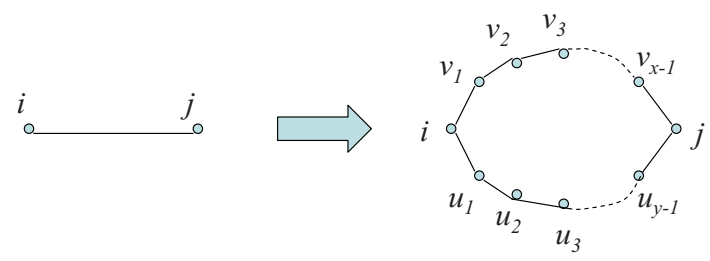

Figure 4. An illustration of labels of nodes in an $(x, y)$-flower: each edge connecting two old nodes $i$ and $j$ generates $x+y-2$ new nodes $v_{1}, v_{2}, \ldots$, $v_{x-1}, u_{1}, u_{2}, \ldots, u_{y-1}$ (color figure available online).

starting from node $u$ [Lovász 96]. By definition and (3.1), we have

$$
\langle C\rangle_{n}=\frac{1}{N_{n}} \sum_{v \in V_{n}} C_{i_{0} v}^{(n)}=\frac{\sum_{v \in V_{n}} H_{v i_{0}}^{(n)}+\sum_{v \in V_{n}} H_{i_{0} v}^{(n)}}{N_{n}} .
$$

We will first address the evolution of $H_{v i_{0}}^{(n)}$. By the construction rule, an edge connected with an old node $i$ and an old node $j$ at generation $n$ will create $x+$ $y-2$ new nodes at generation $n+1$, among which $x-1$ nodes $v_{1}, v_{2}, \ldots, v_{x-1}$ together with $i$ and $j$ constitute a path of length $x$ edges, and the other $y-1$ nodes $u_{1}, u_{2}, \ldots, u_{y-1}$ together with $i$ and $j$ constitute a path of $y$ edges (see Figure 4). We call $v_{1}$ and $u_{1}$ first-order new neighbors of $i, v_{2}$ and $u_{2}$ secondorder new neighbors of $i$, and so forth.

Consider a random walk on $F_{n+1}(x, y)$. By construction, as the network grows from generation $n$ to $n+1$, the degree of node $i$ at generation $n$, denoted by $k_{i}^{(n)}$, increases from $k_{i}^{(n)}$ to $k_{i}^{(n+1)}=2 k_{i}^{(n)}$. Among these $2 k_{i}^{(n)}$ neighbors, half are the first-order new neighbors of $i$ belonging to paths of $x$ edges, and the other half are the first-order new neighbors of $i$ belonging to paths of $y$ edges. Let $Z$ be the hitting time commencing from node $i$ to any of its $k_{i}^{(n)}$ old neighbors, say $j$, namely, those nodes adjacent to node $i$ at generation $n$. Let $X_{1 s}\left(1 \leq s \leq k_{i}^{(n)}\right)$ be the hitting time starting at any of $i$ 's $k_{i}^{(n)}$ first-order new neighbors belonging to a path of $x$ edges to node $j$, and similarly, let $Y_{1 s}\left(1 \leq s \leq k_{i}^{(n)}\right)$ be the hitting time starting at any of $i$ 's $k_{i}^{(n)}$ first-order new neighbors belonging to a path of $y$ edges to node $j$. Then

$$
Z=\frac{1}{2 k_{i}^{(n)}} \sum_{s=1}^{k_{i}^{(n)}}\left(1+X_{1 s}\right)+\frac{1}{2 k_{i}^{(n)}} \sum_{s=1}^{k_{i}^{(n)}}\left(1+Y_{1 s}\right) .
$$


Define $X_{1}=\sum_{s=1}^{k_{i}^{(n)}} X_{1 s} / k_{i}^{(n)}$ and $Y_{1}=\sum_{s=1}^{k_{i}^{(n)}} Y_{1 s} / k_{i}^{(n)}$. Hence (3.13) can be rewritten in the compact form

$$
Z=\frac{1+X_{1}}{2}+\frac{1+Y_{1}}{2}
$$

Analogously, we can define $X_{t}(2 \leq t \leq x-1)$ and $Y_{l}(2 \leq l \leq y-1)$. Together with (3.13), we have the following backward equations:

$$
\begin{aligned}
Z & =\frac{1}{2}\left(1+X_{1}\right)+\frac{1}{2}\left(1+Y_{1}\right), \\
X_{1} & =\frac{1}{2}(1+Z)+\frac{1}{2}\left(1+X_{2}\right), \\
X_{2} & =\frac{1}{2}\left(1+X_{1}\right)+\frac{1}{2}\left(1+X_{3}\right), \\
& \cdots \\
X_{x-1} & =\frac{1}{2}+\frac{1}{2}\left(1+X_{x-2}\right), \\
Y_{1} & =\frac{1}{2}(1+Z)+\frac{1}{2}\left(1+Y_{2}\right), \\
Y_{2} & =\frac{1}{2}\left(1+Y_{1}\right)+\frac{1}{2}\left(1+Y_{3}\right), \\
& \cdots \\
Y_{y-1} & =\frac{1}{2}+\frac{1}{2}\left(1+Y_{y-2}\right) .
\end{aligned}
$$

Eliminating $X_{t}(1 \leq t \leq x-1)$ and $Y_{l}(1 \leq l \leq y-1)$ from (3.14), we obtain $Z=x y$. Hence, on the growth of $(x, y)$-flowers from generation $n$ to generation $n+1$, the hitting time between node $i$ and any one of the old nodes $j$ (i.e., $i, j \in$ $\left.F_{n}(x, y)\right)$ increases by the factor $x y$. This is because the hitting time between any two adjacent nodes at generation $n$ increases from 1 to $x y$ at generation $n+1$. Thus we have

$$
H_{v i_{0}}^{(n+1)}=x y H_{v i_{0}}^{(n)}=w H_{v i_{0}}^{(n)} .
$$

Let $\tilde{V}_{n}$ be the set of nodes in $F_{n}(x, y)$ that enter the network at generation $n$. Hence, we have $V_{n}=\tilde{V}_{n} \cup V_{n-1}$. For $1 \leq s \leq n$, we define the following quantities:

$$
H_{s}^{(n)}=\sum_{v \in V_{s}} H_{v i_{0}}^{(n)}
$$

and

$$
\tilde{H}_{s}^{(n)}=\sum_{v \in \tilde{V}_{s}} H_{v i_{0}}^{(n)} .
$$


Using (3.15), we observe that $H_{n}^{(n)}$ obeys the following recurrence relation:

$$
H_{n}^{(n)}=H_{n-1}^{(n)}+\tilde{H}_{n}^{(n)}=w H_{n-1}^{(n-1)}+\tilde{H}_{n}^{(n)} .
$$

To solve $H_{n}^{(n)}$, we need first to figure out $\tilde{H}_{n}^{(n)}$.

According to the construction algorithm, for a given edge incident on two nodes $i$ and $j$ in $F_{n}(x, y)$, it will give rise to the creation of $x+y-2$ new nodes, say $v_{1}, \ldots, v_{x-1}, u_{1}, \ldots, u_{y-1}$, at generation $n+1$ (cf. Figure 4 ). The hitting times for these new nodes satisfy relations

$$
\begin{aligned}
H_{v_{1} i_{0}}^{(n+1)} & =\frac{1}{2}\left(1+H_{i i_{0}}^{(n+1)}\right)+\frac{1}{2}\left(1+H_{v_{2} i_{0}}^{(n+1)}\right), \\
H_{v_{2} i_{0}}^{(n+1)} & =\frac{1}{2}\left(1+H_{v_{1} i_{0}}^{(n+1)}\right)+\frac{1}{2}\left(1+H_{v_{3} i_{0}}^{(n+1)}\right), \\
& \cdots \\
H_{v_{x-1} i_{0}}^{(n+1)} & =\frac{1}{2}\left(1+H_{v_{x-2} i_{0}}^{(n+1)}\right)+\frac{1}{2}\left(1+H_{j i_{0}}^{(n+1)}\right), \\
H_{u_{1} i_{0}}^{(n+1)} & =\frac{1}{2}\left(1+H_{i i_{0}}^{(n+1)}\right)+\frac{1}{2}\left(1+H_{u_{2} i_{0}}^{(n+1)}\right), \\
H_{u_{2} i_{0}}^{(n+1)} & =\frac{1}{2}\left(1+H_{u_{1} i_{0}}^{(n+1)}\right)+\frac{1}{2}\left(1+H_{u_{3} i_{0}}^{(n+1)}\right), \\
& \cdots \\
H_{u_{y}-1}^{(n+1)} i_{0} & =\frac{1}{2}\left(1+H_{u_{y}-2}^{(n+1)}\right)+\frac{1}{2}\left(1+H_{j i_{0}}^{(n+1)}\right) .
\end{aligned}
$$

Arguing similarly as in [Zhang et al. 09, Zhang et al. 11], we can obtain the following recurrence relation from (3.19):

$$
\tilde{H}_{n+2}^{(n+2)}=w z \tilde{H}_{n+1}^{(n+1)}+\frac{1}{6}\left(z^{2}-3 w-1\right)(z-2 w) z^{n+1} .
$$

Using the initial condition $\tilde{H}_{1}^{(1)}=\left(z^{3}-z\right) / 6-w$, we solve (3.20) inductively to get

$$
\begin{aligned}
\tilde{H}_{n}^{(n)}= & \left(\frac{\left(z^{2}-3 w-1\right)(z-2 w)}{6(w-1)}+\frac{z^{3}-z}{6}-w\right)(w z)^{n-1} \\
& -\frac{\left(z^{2}-3 w-1\right)(z-2 w) z^{n}}{w-1} .
\end{aligned}
$$

Substituting (3.21) into (3.18), we arrive at

$$
H_{n}^{(n)}=\frac{A_{1} z^{n}}{w-z}+A_{3} w^{n}+\frac{A_{2} z^{n} w^{n-1}}{z-1},
$$


by involving the initial condition $H_{1}^{(1)}=\left(z^{3}-z\right) / 6$, where

$$
\begin{aligned}
& A_{1}=\frac{\left(z^{2}-3 w-1\right)(z-2 w)}{6(w-1)} \\
& A_{2}=A_{1}+\frac{z^{3}-z}{6}-w \\
& A_{3}=\frac{z^{3}-z}{6 w}-\frac{A_{1} z}{w(w-z)}-\frac{A_{2} z}{w(z-1)} .
\end{aligned}
$$

Next, we need to study the evolution of the analogous hitting time $H_{i_{0} v}^{(n)}$. Note that a random walk originating at $i_{0}$ and ending at node $v_{1}$ must pass either node $i$ or node $v_{2}$; cf. Figure 3 . A similar consideration reveals that the analogue of relation (3.19) still holds. The counterparts for the initial conditions $H_{1}^{(1)}$ and $\tilde{H}_{1}^{(1)}$ are unaltered, since $F_{1}(x, y)$ is a symmetric cycle graph. Hence, although $H_{i_{0} v}^{(n)}$ is not equal to $H_{v i_{0}}^{(n)}$ in general, we have $\sum_{v \in V_{s}} H_{i_{0} v}^{(n)}=H_{n}^{(n)}$. Thus, it follows from (3.12), (3.16), and (3.22) that

$$
\langle C\rangle_{n}=\frac{2 H_{n}^{(n)}}{N_{n}}=\frac{2 A_{1} z^{n}}{w-z}+2 A_{3} w^{n}+\frac{2 A_{2} z^{n} w^{n-1}}{z-1},
$$

where $A_{1}, A_{2}$, and $A_{3}$ are given as before.

From (2.2), we have that $z^{n}=(z-1) N_{n} /(z-2)-z /(z-2)$. Inserting this into (3.23), we obtain, after some rearrangement,

$$
\begin{aligned}
\langle C\rangle_{n}= & \frac{2 A_{1}}{(w-z) N_{n}}\left(\frac{z-1}{z-2} N_{n}-\frac{z}{z-2}\right)+\frac{2 A_{3}}{N_{n}}\left(\frac{z-1}{z-2} N_{n}-\frac{z}{z-2}\right)^{\ln w / \ln z} \\
& +\frac{2 A_{2}}{w(z-1) N_{n}}\left(\frac{z-1}{z-2} N_{n}-\frac{z}{z-2}\right)^{\ln w z / \ln z}
\end{aligned}
$$

Note that $w>1$ and $z>2$, and some simplifications of (3.24) lead to

$$
\langle C\rangle_{n} \sim \frac{2 A_{2}}{w(z-2)} N_{n}^{\ln w / \ln z}=\frac{z^{2}-4}{3(w-1)} N_{n}^{\ln w / \ln z},
$$

as $n \rightarrow \infty$.

In Figure 5, we compare the asymptotics of the mean commute time in (3.2) with numerical values calculated from the fundamental matrix [Kemeny and Snell 76] of random walks on $F_{n}(2,3)$ (simply double the mean first-passage time, similarly as in [Zhang et al. 09]). We observe that the asymptotic results are in perfect agreement with numerical results for all $3 \leq n \leq 6$. The consistency confirms that Theorem 3.1 is valid. 


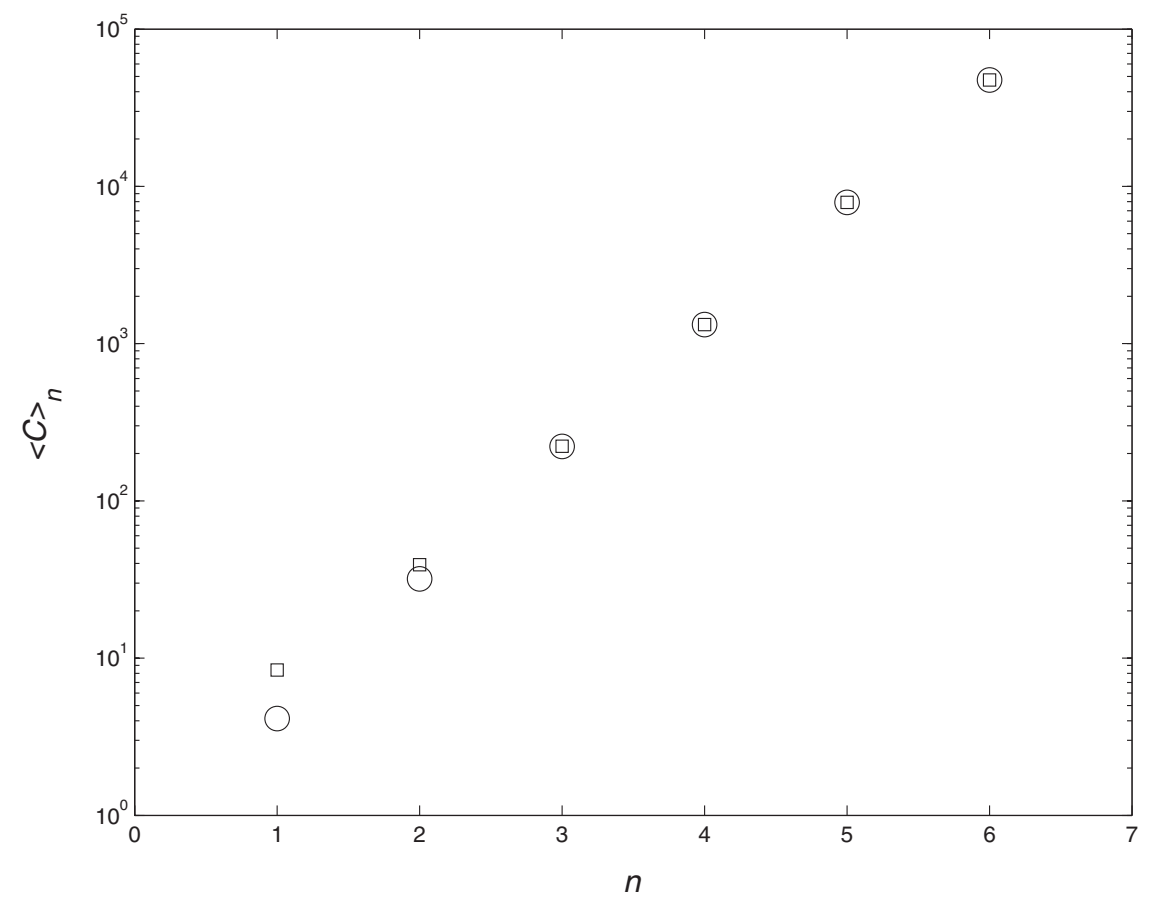

Figure 5. Mean commute time $\langle C\rangle_{n}$ versus generation $n$ for random walks on $F_{n}(x, y)$ with $x=2$ and $y=3$. The squares are theoretical asymptotics from (3.2), while the circles correspond to the numerical calculations from the fundamental matrix.

\section{Conclusion}

We have studied simple random walks on a class of deterministic scale-free networks, called $(x, y)$-flowers. Networks in this family are self-similar and hierarchical, in the sense that each network contains $x+y$ subgraphs that resemble the whole, which advantageously makes them amenable to analytical treatment. We focus on the mean commute time between an initial node (i.e., hub node) and other nodes in the networks. We derive exact and asymptotic expressions for mean commute time and show that it grows as a power-law function of the network size with exponent governed by the parameters $x$ and $y$ in a delicate manner.

Based on a connection between commute time and effective resistance, we determine the mean effective resistance between an initial node and other nodes in $(x, y)$-flowers. It is shown to change sharply from zero to infinity between 
different combinations of parameters. We also compare the mean commute times for $(x, y)$-flowers with those for Erdős-Rényi random graphs. The mean commute times for $(x, y)$-flowers with finite fractal dimensions (i.e., $x>1$ ) are shown to be even almost surely greater than those for sparse Erdős-Rényi graphs with a logarithmically diverging mean degree. In addition, an asymptotic upper bound for the mean commute time in terms of average degree of the network is derived $($ as $n \rightarrow \infty)$.

There remain many interesting open problems. For example, since we consider only mean commute times involving one of the hubs, it would be desirable to study commute time between general nodes. Exploration of cover time and of mixing time on the $(x, y)$-flowers would be more challenging (but certainly interesting) research problems. On the other hand, the $(x, y)$-flowers have small-world effects and fractal behaviors, while Erdős-Rényi random graphs do not. Another future direction might be to improve our understanding of the role of local clustering and fractal dimension on the mean commute time. This should help in finding efficient network algorithms.

\section{References}

[Barabási and Albert 99] A.-L. Barabási and R. Albert. "Emergence of Scaling in Random Networks." Science 286 (1999), 509-512.

[Bartumeus and Levin 08] F. Bartumeus and S. A. Levin. "Fractal Reorientation Clocks: Linking Animal Behavior to Statistical Patterns of Search." Proc. Natl. Acad. Sci. USA 105 (2008), 19072-19077.

[Bentz et al. 10] J. L. Bentz, J. W. Turner, and J. J. Kozak. "Analytic Expression for the Mean Time to Absorption for a Random Walker on the Sierpiński Gasket. II. The eigenvalue spectrum." Phys. Rev. E, 82 (2010), 011137.

[Bollobás and Riordan 04] B. Bollobás and O. Riordan. "Robustness and Vulnerability of Scale-Free Random Graphs." Internet Math. 1 (2004), 1-35.

[Brin and Page 98] S. Brin and L. Page. "The Anatomy of a Large-Scale Hypertextual Web Search Engine." Computer Networks and ISDN Systems 30 (1998), 107-117.

[Burioni and Cassi 05] R. Burioni and D. Cassi. "Random Walks on Graphs: Ideas, Techniques and Results." J. Phys. A: Math. Gen. 38 (2005), R45-R78.

[Candia et al. 07] J. Candia, P. E. Parris, and V. M. Kenkre, "Transport Properties of Random Walks on Scale-Free/Regular-Lattice Hybrid Networks." J. Stat. Phys. 129 (2007), 323-333.

[Chandra et al. 96] A. K. Chandra, P. Raghavan, W. L. Ruzzo, R. Smolensky, and P. Tiwari. "The Electrical Resistance of a Graph Captures Its Commute and Cover Times." Comput. Complexity 6 (1996), 312-340. 
[Chu et al. 10] X. Chu, Z. Zhang, J. Guan, S. Zhou, and M. Li. "Different Behaviors of Epidemic Spreading in Scale-Free Networks with Identical Degree Sequence." J. Phys. A: Math. Theor. 43 (2010), 065001.

[Codling et al. 08] E. A. Codling, M. J. Plank, and S. Benhamou. "Random Walk Models in Biology." J. R. Soc. Interface 5 (2008), 813-834.

[Dorogovtsev et al. 02] S. N. Dorogovtsev, A. V. Goltsev, and J. F. F. Mendes. "Pseudofractal Scale-Free Web." Phys. Rev. E 65 (2002), 066122.

[Doyle and Snell 84] P. G. Doyle and J. L. Snell. Random Walks and Electrical Networks. Mathematical Association of America, 1984.

[Esfandiar et al. 10] P. Esfandiar, F. Bonchi, D. F. Gleich, C. Greif, L. V. S. Lakshmanan, and B.-W. On. "Fast Katz and Commuters: Efficient Estimation of Social Relatedness in Large Networks." In Algorithms and Models for the Web Graph, Lecture Notes in Computer Science 6516, pp. 132-145. Springer-Verlag, 2010.

[Fouss et al. 07] F. Fouss, A. Pirotte, J.-M. Renders, and M. Saerens. "Random-Walk Computation of Similarities between Nodes of a Graph with Application to Collaborative Recommendation." IEEE T. Knowl. Data En. 19 (2007), 355-369.

[Gallos 04] L. K. Gallos. "Random Walk and Trapping Processes on Scale-Free Networks." Phys. Rev. E 70 (2004), 046116.

[García Cantú and Abad 08] A. García Cantú and E. Abad. "Efficiency of Trapping Processes in Regular and Disordered Networks." Phys. Rev. E 77 (2008), 031121.

[Gori et al. 04] M. Gori, M. Maggini, and L. Sarti. "Graph Matching Using Random Walks." In Proc. 17th International Conference on Pattern Recognition, Cambridge, UK, vol. 3, pp. 394-397, 2004.

[Hattori et al. 94] K. Hattori, T. Hattori, and H. Watanabe. "Asymptotically OneDimensional Diffusions on the Sierpiński Gasket and the abc-Gaskets." Probab. Theory Relat. Fields 100 (1994), 85-116.

[Hinczewski and Berker 06] M. Hinczewski and A. N. Berker. "Inverted BerezinskiiKosterlitz-Thouless Singularity and High-Temperature Algebraic Order in an Ising Model on a Scale-Free Hierarchical-Lattice Small-World Network." Phys. Rev. E 73 (2006), 066126.

[Hruz and Peter 11] T. Hruz and U. Peter. "Nongrowing Preferential Attachment Random Graphs." Internet Math. 6 (2011), 461-487.

[Kemeny and Snell 76] J. G. Kemeny and J. L. Snell. Finite Markov Chains. Springer, 1976.

[Lovász 96] L. Lovász. "Random Walks on Graphs: a Survey." In Combinatorics, Paul Erdős Is Eighty, vol. 2, pp. 353-397. János Bolyai Mathematical Society, 1996.

[Maiya and Berger-Wolf 10] A. S. Maiya and T. Y. Berger-Wolf. "Expansion and Search in Networks." In Proc. 19th ACM International Conference on Information and Knowledge Management, Toronto, Canada, pp. 239-248, 2010.

[Meila and Shi 01] M. Meila and J. Shi. "A Random Walks View of Spectral Segmentation." In Proc. 8th International Workshop on Artificial Intelligence and Statistics, Key West, Florida, pp. 92-97, 2001. 
[Metzler and Klafter 00] R. Metzler and J. Klafter. "The Random Walk's Guide to Anomalous Diffusion: A Fractional Dynamics Approach." Phys. Rep. 339 (2000), $1-77$.

[Molloy and Reed 95] M. Molloy and B. Reed. "A Critical Point for Random Graphs with a Given Degree Sequence." Random Struct. Algorithms 6 (1995), 161180 .

[Montroll 56] E. W. Montroll. "Random Walks in Multidimensional Spaces, Especially on Periodic Lattices." SIAM J. Appl. Math. 4 (1956), 241-260.

[Newman et al. 06] M. Newman, A.-L. Barabási, and D. J. Watts. The Structure and Dynamics of Networks. Princeton University Press, 2006.

[Noh and Kim 06] J. D. Noh and S.-W. Kim. "Random-Walk and Pair-Annihilation Processes on Scale-Free Networks." J. Korean Phys. Soc. 48 (2006), S202-S207.

[Palacios 09] J. L. Palacios. "On Hitting Times of Random Walks on Trees." Stat. Prob. Lett. 79 (2009), 234-236.

[Polynikis 06] A. Polynikis. "Random Walks and Scale-Free Networks." M.Sc. thesis, University of York, UK, 2006.

[Qiu and Hancock 07] H. Qiu and E. R. Hancock. "Clustering and Embedding Using Commute Times." IEEE Trans. Pattern Anal. Mach. Intell. 29 (2007), 1873-1890.

[Rozenfeld and ben-Avraham 07] H. D. Rozenfeld and D. ben-Avraham. "Percolation in Hierarchical Scale-Free Nets." Phys. Rev. E 75 (2007), 061102.

[Rozenfeld et al. 07] H. D. Rozenfeld, S. Havlin, and D. ben-Avraham. "Fractal and Transfractal Recursive Scale-Free Nets." New J. Phys. 9 (2007), 175.

[Shang 10] Y. Shang. "Multi-agent Coordination in Directed Moving Neighborhood Random Networks." Chin. Phys. B 19 (2010), 070201.

[Shang 12] Y. Shang. "Percolation in a Hierarchical Lattice." Z. Naturforsch. A 67 (2012), 225-229.

[Sood et al. 05] V. Sood, S. Redner, and D. ben-Avraham. "First-Passage Properties of the Erdős-Rényi Random graph." J. Phys. A: Math. Gen. 38 (2005), 109123 .

[Tanaka et al. 12] G. Tanaka, K. Morino, and K. Aihara. "Dynamical Robustness in Complex Networks: The Crucial Role of Low-Degree Nodes." Sci. Rep. 2 (2012), 232 .

[Tejedor et al. 11] V. Tejedor, O. Bénichou, and R. Voituriez. "Close or Connected: Distance and Connectivity Effects on Transport in Networks." Phys. Rev. E 83 (2011), 066102.

[Turk 01] M. Turk. "A Random Walk through Eigenspace." IEICE Trans. Inf. Syst., E84-D (2001), 1586-1595.

[Weiss 94] G. H. Weiss. Aspects and Applications of the Random Walk. North-Holland, 1994.

[Yen et al. 07] L. Yen, F. Fouss, C. Decaestecker, P. Francq, and M. Saerens. "Graph Nodes Clustering Based on the Commute-Time Kernel." In Advances in Knowledge Discovery and Data Mining, Lecture Notes in Computer Science 4426, pp. 1037-1045. Springer-Verlag, 2007. 
[Yun et al. 09] C.-K. Yun, B. Kahng, and D. Kim. "Annihilation of Two-Species Reaction-Diffusion Processes on Fractal Scale-Free Networks." New J. Phys. 11 (2009), 063025.

[Zhang et al. 09] Z. Zhang, W. Xie, S. Zhou, M. Li, and J. Guan. "Distinct Scalings for Mean First-Passage Time of Random Walks on Scale-Free Networks with the Same Degree Sequence." Phys. Rev. E 80 (2009), 061111.

[Zhang et al. 11] Z. Zhang, Y. Yang, and S. Gao. "Role of Fractal Dimension in Random Walks on Scale-Free Networks." Eur. Phys. J. B 84 (2011), 331-338.

Yilun Shang, Institute for Cyber Security, University of Texas at San Antonio, San Antonio, Texas 78249 (shylmath@hotmail.com) 